\title{
Stochastic Volterra integral equations with a parameter
}

\section{Yanqing Wang*}

\section{"Correspondence:}

yqwang@amss.ac.cn

School of Mathematics and

Statistics, Southwest University,

Chongqing, 400715, China

\begin{abstract}
In this paper, we study the properties of continuity and differentiability of solutions to stochastic Volterra integral equations and backward stochastic Volterra integral equations depending on a parameter.
\end{abstract}

MSC: $60 \mathrm{H} 20 ; 65 \mathrm{C} 30$

Keywords: stochastic Volterra integral equation; backward stochastic Volterra integral equation; parameter; continuity; differentiability

\section{Introduction and preliminaries}

Let $(\Omega, \mathcal{F}, \mathbb{F}, \mathbb{P})$ be a complete filtered probability space on which a $d$-dimensional standard Brownian motion $W(\cdot)$ is defined such that $\mathbb{F} \equiv\left\{\mathcal{F}_{t}\right\}_{t \geq 0}$ is its natural filtration augmented by all the $\mathbb{P}$-null sets.

In this paper, for given $T>0$, we consider the continuity and differentiability of solutions to the following stochastic Volterra integral equations (SVIEs) with respect to a parameter $\alpha$ :

$$
X_{\alpha}(t)=\varphi_{\alpha}(t)+\int_{0}^{t} b_{\alpha}\left(t, s, X_{\alpha}(s)\right) d s+\int_{0}^{t} \sigma_{\alpha}\left(t, s, X_{\alpha}(s)\right) d W(s), \quad t \in[0, T]
$$

and backward stochastic Volterra integral equations (BSVIEs) with respect to $\alpha$ :

$$
\begin{aligned}
Y_{\alpha}(t)= & \psi_{\alpha}(t)+\int_{t}^{T} f_{\alpha}\left(t, s, Y_{\alpha}(s), Z_{\alpha}(t, s), Z_{\alpha}(s, t)\right) d s \\
& +\int_{t}^{T} Z_{\alpha}(t, s) d W(s), \quad t \in[0, T] .
\end{aligned}
$$

Here $\alpha \in \mathbb{R}$ and, for any given $\alpha, \varphi_{\alpha}:[0, T] \times \Omega \rightarrow \mathbb{R}^{m}, b_{\alpha}:[0, T]^{2} \times \mathbb{R}^{m} \rightarrow \mathbb{R}^{m}, \sigma_{\alpha} \equiv$ $\left(\sigma_{\alpha}^{1}, \sigma_{\alpha}^{2}, \ldots, \sigma_{\alpha}^{d}\right):[0, T]^{2} \times \mathbb{R}^{m} \rightarrow \mathbb{R}^{m \times d}, f_{\alpha}:[0, T]^{2} \times \mathbb{R}^{n} \times \mathbb{R}^{n \times d} \times \mathbb{R}^{n \times d} \rightarrow \mathbb{R}^{n}$ are Borel measurable functions.

The theory of stochastic differential equations (SDEs), including SDEs with parameters, is an important topic in stochastic processes. There exists much research work on this topic (see, e.g., [1-4]). SVIEs, as a natural and nontrivial extensions of SDEs, not only have their distinctive features (e.g., containing memories), but they also have interesting applications such as in stochastic control (see, e.g., $[5,6])$. The theory for SVIEs is much richer 
than that of SDEs. SVIEs with regular kernels and driven by Brownian motions were first studied in the 1970s and early-1980s (see, e.g., [7-9]). Later, Protter in [10] studied SVIEs driven by general semimartingales. BSVIEs also can be viewed generalizations of BSDEs, first studied by Lin ([11]); and then deeply investigated by Yong ([12]).

Building on previous work concerning SDEs and BSDEs with a parameter $([1-4,13])$, this article is devoted to studying properties of solutions to SVIE (1.1) and BSVIE (1.2) with respect to a parameter, mainly continuity and differentiability. For the continuity of solutions to SVIEs with respect to a parameter, the reader can refer to [7, 8]; and for the differentiability the reader can refer to [14]. In this work, we presents more general and complete results on this topic.

We now introduce some basic notations. Let $H=\mathbb{R}^{n}, \mathbb{R}^{n \times m}$, etc.

- $L_{\mathcal{F}_{T}}^{2}(\Omega ; H)$ is the set of all $\mathcal{F}_{T}$-measurable random variables $\xi$ valued in $H$ such that

$$
\|\xi\|_{L_{\mathcal{F}_{T}}^{2}(\Omega ; H)} \equiv\left(\mathbb{E}|\xi|^{2}\right)^{\frac{1}{2}}<\infty
$$

- $L_{\mathbb{F}}^{2}(\Omega \times(0, T) ; H)$ is the set of all $\mathbb{F}$-progressively measurable processes $\varphi(\cdot)$ valued in $H$ such that

$$
\|\varphi(\cdot)\|_{L_{\mathbb{F}}^{2}(\Omega \times(0, T) ; H)} \equiv\left[\mathbb{E} \int_{0}^{T}|\varphi(t)|^{2} d t\right]^{\frac{1}{2}}<\infty .
$$

- $C_{\mathbb{F}}\left([0, T] ; L^{2}(\Omega ; H)\right)$ is the set of all $\mathbb{F}$-progressively measurable processes $\varphi(\cdot)$ valued in $H$ such that, for almost all $\omega \in \Omega, t \mapsto \varphi(t, \omega)$ is continuous and

$$
\|\varphi(\cdot)\|_{C_{\mathbb{F}}\left([0, T] ; L^{2}(\Omega ; H)\right)} \equiv\left[\sup _{t \in[0, T]} \mathbb{E}\left(|X(t)|^{2}\right)\right]^{\frac{1}{2}}<\infty .
$$

- $L^{2}\left(0, T ; L_{\mathbb{F}}^{2}(\Omega \times(0, T) ; H)\right)$ is the set of all processes $Z:[0, T]^{2} \times \Omega \rightarrow H$ such that, for almost $t \in[0, T], Z(t, \cdot) \in L_{\mathbb{F}}^{2}(\Omega \times(0, T) ; H)$ and

$$
\|Z(\cdot, \cdot)\|_{L^{2}\left(0, T ; L_{\mathbb{F}}^{2}(\Omega \times(0, T) ; H)\right)} \equiv\left[\mathbb{E} \int_{0}^{T} \int_{0}^{T}|Z(t, s)|^{2} d s d t\right]^{\frac{1}{2}}<\infty .
$$

- $\mathcal{H}^{2}[0, T] \equiv L_{\mathbb{F}}^{2}\left(\Omega \times(0, T) ; \mathbb{R}^{n}\right) \times L^{2}\left(0, T ; L_{\mathbb{F}}^{2}\left(\Omega \times(0, T) ; \mathbb{R}^{n \times d}\right)\right)$.

In what follows, we will make use of the following elementary assumptions:

(A1) For any $\alpha \in \mathbb{R}, \varphi_{\alpha}(\cdot)$ is an $\mathbb{F}$-adapted continuous process, and there exists a positive constant $L$ (independently on $\alpha$ ), such that

$$
\begin{aligned}
& \sup _{0 \leq t \leq T} \mathbb{E}\left|\varphi_{\alpha}(t)\right|^{2} \leq L^{2}, \\
& \left|b_{\alpha}(t, s, x)-b_{\alpha}(t, s, y)\right|+\left|\sigma_{\alpha}(t, s, x)-\sigma_{\alpha}(t, s, y)\right| \leq L|x-y|, \\
& \quad t, s \in[0, T], x, y \in \mathbb{R}^{d},
\end{aligned}
$$

and

$$
\left|b_{\alpha}(\cdot, \cdot, 0)\right|+\left|\sigma_{\alpha}(\cdot, \cdot, 0)\right| \leq L
$$


(A2) There exists a positive constant $L$, such that, for any $\alpha \in \mathbb{R}$,

$$
\begin{aligned}
& \sup _{0 \leq t \leq T} \mathbb{E}\left|\psi_{\alpha}(t)\right|^{2} \leq L^{2}, \\
& \left|f_{\alpha}\left(t, s, y_{1}, z_{1}, v_{1}\right)-f_{\alpha}\left(t, s, y_{2}, z_{2}, v_{2}\right)\right| \leq L\left(\left|y_{1}-y_{2}\right|+\left|z_{1}-z_{2}\right|+\left|v_{1}-v_{2}\right|\right), \\
& \quad t, s \in[0, T], y_{1}, y_{2} \in \mathbb{R}^{m}, z_{1}, z_{2}, v_{1}, v_{2} \in \mathbb{R}^{m \times d},
\end{aligned}
$$

and

$$
\left|f_{\alpha}(\cdot, \cdot, 0,0,0)\right| \leq L
$$

The rest of this paper is organized as follows. In Section 2, we review the well-posedness and continuity results for SVIEs depending on a parameter, and give the property of solutions' differentiability with respect to that parameter. Section 3 is devoted to studying the continuity and differentiability of solutions to BSVIEs with respect to a parameter.

\section{SVIEs with a parameter}

Firstly, we present the definition of solution to SVIE (1.1).

Definition 2.1 For any $\alpha \in \mathbb{R}$, a stochastic process $X_{\alpha}(\cdot) \in C_{\mathbb{F}}\left([0, T] ; L^{2}\left(\Omega ; \mathbb{R}^{m}\right)\right)$ is called an adapted solution to SVIE (1.1) on $[0, T]$ if (1.1) holds in the usual Itô's sense for all $t \in[0, T]$.

In the following, the well-posedness result comes from [7-9] and the property of continuity of solutions to (1.1) with respect to the parameter $\alpha$ comes from $[7,8]$.

Theorem 2.2 Let assumption (A1) hold. Then, for any $\alpha \in \mathbb{R}$, SVIE (1.1) admits a unique solution $X_{\alpha}(\cdot)$. Furthermore, we have

$$
\begin{aligned}
\sup _{0 \leq t \leq T} \mathbb{E}\left|X_{\alpha}(t)\right|^{2} \leq & C \sup _{0 \leq t \leq T}\left\{\mathbb{E}\left|\varphi_{\alpha}(t)\right|^{2}\right. \\
& \left.+\mathbb{E}\left(\int_{0}^{t}\left|b_{\alpha}(t, s, 0)\right| d s\right)^{2}+\mathbb{E}\left(\int_{0}^{t}\left|\sigma_{\alpha}(t, s, 0)\right|^{2} d s\right)\right\},
\end{aligned}
$$

where $C$ is a constant.

Theorem 2.3 Assume that the coefficients $\varphi_{\alpha}(\cdot), b_{\alpha}(\cdot, \cdot, \cdot), \sigma_{\alpha}(\cdot, \cdot, \cdot)$ in (1.1) satisfy assumption (A1), and

$$
\lim _{\alpha^{\prime} \rightarrow \alpha} \sup _{0 \leq t \leq T} \mathbb{E}\left|\varphi_{\alpha^{\prime}}(t)-\varphi_{\alpha}(t)\right|^{2}=0,
$$

and that, for any $N>0, s, t \in[0, T], s \leq t$ and $\varepsilon>0$,

$$
\lim _{\alpha^{\prime} \rightarrow \alpha} P\left(\sup _{|x|<N}\left(\left|b_{\alpha^{\prime}}(t, s, x)-b_{\alpha}(t, s, x)\right|+\left|\sigma_{\alpha^{\prime}}(t, s, x)-\sigma_{\alpha}(t, s, x)\right|\right)>\varepsilon\right)=0 .
$$

Then

$$
\lim _{\alpha^{\prime} \rightarrow \alpha} \sup _{0 \leq t \leq T} \mathbb{E}\left|x_{\alpha^{\prime}}(t)-x_{\alpha}(t)\right|^{2}=0
$$


Now, we are in the step to study the differentiability of solutions to SVIEs with respect to a parameter. Firstly, we give the definition of derivative of random variables.

Definition 2.4 A random variable $\eta$ is called the derivative of a family of random variables $\left\{\xi_{\alpha}\right\}$ in $L^{2}$-norm if the following holds:

$$
\lim _{\Delta \alpha \rightarrow 0} \mathbb{E}\left|\frac{\xi_{\alpha+\Delta \alpha}-\xi_{\alpha}}{\Delta \alpha}-\eta\right|^{2}=0
$$

For convenience, we denote $\eta$ by $\partial_{\alpha} \xi_{\alpha}$. The following result states the solutions' differentiability with respect to a parameter.

Theorem 2.5 Suppose assumption (A1) hold. Let $X_{\alpha}(\cdot)$ be solution to

$$
X_{\alpha}(t)=\varphi_{\alpha}(t)+\int_{0}^{t} b_{\alpha}\left(t, s, X_{\alpha}(s)\right) d s+\int_{0}^{t} \sigma_{\alpha}\left(t, s, X_{\alpha}(s)\right) d W(s), \quad t \in[0, T]
$$

whereby the following conditions are satisfied:

(1) $\partial_{\alpha} \varphi_{\alpha}(t)$ exists, and

$$
\lim _{\Delta \alpha \rightarrow 0} \sup _{0 \leq t \leq T} \mathbb{E}\left|\partial_{\alpha} \varphi_{\alpha}(t)-\frac{1}{\Delta \alpha}\left(\varphi_{\alpha+\Delta \alpha}(t)-\varphi_{\alpha}(t)\right)\right|^{2} \rightarrow 0 ;
$$

(2) $\partial_{\alpha} b_{\alpha}(t, s, x)$ and $\partial_{\alpha} \sigma_{\alpha}(t, s, x)$ exist, and

$$
\begin{gathered}
\lim _{\Delta \alpha \rightarrow 0} \mathbb{E} \int_{0}^{T}\left\{\left|\frac{b_{\alpha+\Delta \alpha}\left(t, s, X_{\alpha}(s)\right)-b_{\alpha}\left(t, s, X_{\alpha}(s)\right)}{\Delta \alpha}-\partial_{\alpha} b_{\alpha}\left(t, s, X_{\alpha}(s)\right)\right|^{2}\right. \\
\left.+\left|\frac{\sigma_{\alpha+\Delta \alpha}\left(t, s, X_{\alpha}(s)\right)-\sigma_{\alpha}\left(t, s, X_{\alpha}(s)\right)}{\Delta \alpha}-\partial_{\alpha} \sigma_{\alpha}\left(t, s, X_{\alpha}(s)\right)\right|^{2}\right\} d s=0 ;
\end{gathered}
$$

(3) $\partial_{x} b_{\alpha}(t, s, x)$ and $\partial_{x} \sigma_{\alpha}(t, s, x)$ exist and are continuous with respect to all arguments, and for some $K$

$$
P\left(\left|\partial_{x} b_{\alpha}(t, s, x)\right| \leq K\right)=1, \quad P\left(\left|\partial_{x} \sigma_{\alpha}^{i}(t, s, x)\right| \leq K\right)=1, \quad i=1,2, \ldots, d
$$

Then $X_{\alpha}(\cdot)$ is differentiable with respect to $\alpha$, and $\partial_{\alpha} X_{\alpha}(\cdot)$, the derivative of $X_{\alpha}(\cdot)$ with respect to $\alpha$, solves the following SVIE:

$$
\begin{aligned}
\partial_{\alpha} X_{\alpha}(t)= & \partial_{\alpha} \varphi_{\alpha}(t)+\int_{0}^{t}\left[\partial_{x} b_{\alpha}\left(t, s, X_{\alpha}(s)\right) \partial_{\alpha} X_{\alpha}(s)+\partial_{\alpha} b_{\alpha}\left(t, s, X_{\alpha}(s)\right)\right] d s \\
& +\int_{0}^{t}\left[\left\langle\partial_{x} \sigma_{\alpha}\left(t, s, X_{\alpha}(s)\right), \partial_{\alpha} X_{\alpha}(s)\right\rangle+\partial_{\alpha} \sigma_{\alpha}\left(t, s, X_{\alpha}(s)\right)\right] d W(s), \quad t \in[0, T],
\end{aligned}
$$

where $\left\langle\partial_{x} \sigma_{\alpha}, \partial_{\alpha} X_{\alpha}\right\rangle=\left(\partial_{x} \sigma_{\alpha}^{i} \partial_{\alpha} X_{\alpha}\right)_{1 \leq i \leq d}$. 
Proof For notational convenience, we can assume that $m=d=1$. Denote $X_{\alpha, \Delta \alpha}(\cdot) \equiv$ $\frac{1}{\Delta \alpha}\left(X_{\alpha+\Delta \alpha}(\cdot)-X_{\alpha}(\cdot)\right)$. Then this stochastic process satisfies

$$
\begin{aligned}
X_{\alpha, \Delta \alpha}(t)= & \frac{\varphi_{\alpha+\Delta \alpha}(t)-\varphi_{\alpha}(t)}{\Delta \alpha} \\
& +\int_{0}^{t} \frac{b_{\alpha+\Delta \alpha}\left(t, s, X_{\alpha+\Delta \alpha}(s)\right)-b_{\alpha}\left(t, s, X_{\alpha}(s)\right)}{\Delta \alpha} d s \\
& +\int_{0}^{t} \frac{\sigma_{\alpha+\Delta \alpha}\left(t, s, X_{\alpha+\Delta \alpha}(s)\right)-\sigma_{\alpha}\left(t, s, X_{\alpha}(s)\right)}{\Delta \alpha} d W(s), \quad t \in[0, T] .
\end{aligned}
$$

Denote by $X_{\alpha, 0}(\cdot)$ the solution to

$$
\begin{aligned}
X_{\alpha, 0}(t)= & \partial_{\alpha} \varphi_{\alpha}(t)+\int_{0}^{t} \partial_{x} b_{\alpha}\left(t, s, X_{\alpha}(s)\right) X_{\alpha, 0}(s) d s+\int_{0}^{t} \partial_{x} \sigma_{\alpha}\left(t, s, X_{\alpha}(s)\right) X_{\alpha, 0}(s) d W(s) \\
& +\int_{0}^{t} \partial_{\alpha} b_{\alpha}\left(t, s, X_{\alpha}(s)\right) d s+\int_{0}^{t} \partial_{\alpha} \sigma_{\alpha}\left(t, s, X_{\alpha}(s)\right) d W(s), \quad t \in[0, T] .
\end{aligned}
$$

To simplify the above two equations, we introduce the following functions: for $\Delta \alpha \neq 0$,

$$
\begin{aligned}
& \tilde{\varphi}_{\Delta \alpha}(t) \equiv \frac{\varphi_{\alpha+\Delta \alpha}(t)-\varphi_{\alpha}(t)}{\Delta \alpha}+\int_{0}^{t} \frac{b_{\alpha+\Delta \alpha}\left(t, s, X_{\alpha}(s)\right)-b_{\alpha}\left(t, s, X_{\alpha}(s)\right)}{\Delta \alpha} d s \\
&+\int_{0}^{t} \frac{\sigma_{\alpha+\Delta \alpha}\left(t, s, X_{\alpha}(s)\right)-\sigma_{\alpha}\left(t, s, X_{\alpha}(s)\right)}{\Delta \alpha} d W(s) \\
& \tilde{b}_{\Delta \alpha}(t, s, x) \equiv \begin{cases}\frac{b_{\alpha+\Delta \alpha}\left(t, s, X_{\alpha+\Delta \alpha}(s)\right)-b_{\alpha+\Delta \alpha}\left(t, s, X_{\alpha}(s)\right)}{X_{\alpha+\Delta \alpha}(s)-X_{\alpha}(s)} x, & X_{\alpha+\Delta \alpha}(s) \neq X_{\alpha}(s) \\
\partial_{x} b_{\alpha+\Delta \alpha}\left(t, s, X_{\alpha}(s)\right) x, & X_{\alpha+\Delta \alpha}(s)=X_{\alpha}(s)\end{cases}
\end{aligned}
$$

for $\Delta \alpha=0$,

$$
\begin{aligned}
& \widetilde{\varphi}_{0}(t) \equiv \partial_{\alpha} \varphi_{\alpha}(t)+\int_{0}^{t} \partial_{\alpha} b_{\alpha}\left(t, s, X_{\alpha}(s)\right) d s+\int_{0}^{t} \partial_{\alpha} \sigma_{\alpha}\left(t, s, X_{\alpha}(s)\right) d W(s) \\
& \widetilde{b}_{0}(t, s, x) \equiv \partial_{x} b_{\alpha}\left(t, s, X_{\alpha}(s)\right) x
\end{aligned}
$$

Similarly, we can define $\widetilde{\sigma}_{\Delta \alpha}(t, s, x)$ and $\widetilde{\sigma}_{0}(t, s, x)$. Using these functions we can express (2.2) and (2.3) in the form

$$
X_{\alpha, \Delta \alpha}(t)=\widetilde{\varphi}_{\Delta \alpha}(t)+\int_{0}^{t} \widetilde{b}_{\Delta \alpha}\left(t, s, X_{\alpha, \Delta \alpha}(s)\right) d s+\int_{0}^{t} \widetilde{\sigma}_{\Delta \alpha}\left(t, s, X_{\alpha, \Delta \alpha}(s)\right) d W(s)
$$

and

$$
X_{\alpha, 0}(t)=\widetilde{\varphi}_{0}(t)+\int_{0}^{t} \widetilde{b}_{0}\left(t, s, X_{\alpha, 0}(s)\right) d s+\int_{0}^{t} \widetilde{\sigma}_{0}\left(t, s, X_{\alpha, 0}(s)\right) d W(s) .
$$

To prove the theorem, it is sufficient to show that

$$
\lim _{\Delta \alpha \rightarrow 0} \mathbb{E}\left|X_{\alpha, \Delta \alpha}(t)-X_{\alpha, 0}(t)\right|^{2}=0
$$

To obtain (2.4), due to Theorem 2.3 , it is only necessary to verify that

$$
\lim _{\Delta \alpha \rightarrow 0} \sup _{0 \leq t \leq T} \mathbb{E}\left|\widetilde{\varphi}_{\Delta \alpha}(t)-\widetilde{\varphi}_{0}(t)\right|^{2}=0
$$


and that, for each $N>0, \varepsilon>0$,

$$
\lim _{\Delta \alpha \rightarrow 0} P\left(\sup _{|x| \leq N}\left(\left|\widetilde{b}_{\Delta \alpha}(t, s, x)-\widetilde{b}_{0}(t, s, x)\right|^{2}+\left|\widetilde{\sigma}_{\Delta \alpha}(t, s, x)-\widetilde{\sigma}_{0}(t, s, x)\right|^{2}\right)>\varepsilon\right)=0 .
$$

Equation (2.6) can be obtained by Condition (3), and (2.5) can be obtained by Condition (1) and Condition (2). That completes the proof.

The next result is an immediate consequence of Theorem 2.5.

Corollary 2.6 Suppose that $X(\cdot)$ solves SVIE:

$$
X(t)=x+\int_{0}^{t} b(t, s, X(s)) d s+\int_{0}^{t} \sigma(t, s, X(s)) d W(s), \quad t \in[0, T]
$$

where $b(\cdot, \cdot, \cdot), \sigma(\cdot, \cdot, \cdot)$ satisfy assumption (A1), and $\partial_{x} b(t, s, x)$ and $\partial_{x} \sigma(t, s, x)$ exist and are continuous with respect to all arguments, and for some $K$

$$
P\left(\left|\partial_{x} b(t, s, x)\right| \leq K\right)=1, \quad P\left(\left|\partial_{x} \sigma^{i}(t, s, x)\right| \leq K\right)=1, \quad i=1,2, \ldots, d
$$

Then $\nabla X(\cdot)\left(\equiv\left(\partial_{x_{i}} X^{j}\right)_{1 \leq i, j \leq m}\right)$ solves the following SVIE:

$$
\begin{aligned}
\nabla X(t)= & I_{m}+\int_{0}^{t} \partial_{x} b(t, s, X(s)) \nabla X(s) d s \\
& +\int_{0}^{t}\left\langle\partial_{x} \sigma(t, s, X(s)), \nabla X(s)\right\rangle d W(s), \quad t \in[0, T]
\end{aligned}
$$

where $I_{m} \in \mathbb{R}^{m \times m}$ is the unit matrix.

\section{BSVIEs with a parameter}

In this section, we consider the continuity and differentiability of solutions to BSVIEs of the form (1.2) with respect to $\alpha$. Firstly, we give the definition of solution to (1.2).

Definition 3.1 Let $S \in[0, T)$. For any $\alpha \in \mathbb{R}$, stochastic processes pair $\left(Y_{\alpha}(\cdot), Z_{\alpha}(\cdot, \cdot)\right) \in$ $\mathcal{H}^{2}[S, T]$ is called an adapted M-solution to BSVIE (1.2) on [S, T] if (1.2) holds in the usual Itô's sense for almost all $t \in[S, T]$ and, in addition, the following holds:

$$
Y_{\alpha}(t)=\mathbb{E}\left(Y_{\alpha}(t) \mid \mathcal{F}_{S}\right)+\int_{S}^{t} Z_{\alpha}(t, s) d W(s), \quad \text { a.e. } t \in[S, T]
$$

The following result comes from [12, Theorem 3.7].

Theorem 3.2 For any $\alpha$, let (A2) hold. Then BSVIE (1.2) admits a unique adapted Msolution $\left(Y_{\alpha}(\cdot), Z_{\alpha}(\cdot, \cdot)\right) \in \mathcal{H}^{2}[0, T]$. Moreover, the following estimate holds:

$$
\begin{aligned}
\left\|\left(Y_{\alpha}(\cdot), Z_{\alpha}(\cdot, \cdot)\right)\right\|_{\mathcal{H}^{2}[S, T]}^{2} & \equiv \mathbb{E}\left\{\int_{S}^{T}\left|Y_{\alpha}(t)\right|^{2} d t+\int_{S}^{T} \int_{S}^{T}\left|Z_{\alpha}(t, s)\right|^{2} d s d t\right\} \\
& \leq C\left(1+\mathbb{E} \int_{S}^{T}\left|\psi_{\alpha}(t)\right|^{2} d t\right), \quad \forall S \in[0, T] .
\end{aligned}
$$


Let $\bar{f}_{\alpha}$ and $\bar{\psi}_{\alpha}$ also satisfy (A2), and $\left(\bar{Y}_{\alpha}(\cdot), \bar{Z}_{\alpha}(\cdot, \cdot)\right) \in \mathcal{H}^{2}[0, T]$ be the adapted M-solution to (1.2) with $f_{\alpha}$ and $\psi_{\alpha}$ replaced by $\bar{f}_{\alpha}$ and $\bar{\psi}_{\alpha}$, respectively. Then there exists a constant $C$ such that, for any $S \in[0, T]$,

$$
\begin{aligned}
& \left\|\left(Y_{\alpha}(\cdot)-\bar{Y}_{\alpha}(\cdot), Z_{\alpha}(\cdot, \cdot)-\bar{Z}_{\alpha}(\cdot, \cdot)\right)\right\|_{\mathcal{H}^{2}[S, T]}^{2} \\
& \leq C \mathbb{E}\left\{\int_{S}^{T}\left|\psi_{\alpha}(t)-\bar{\psi}_{\alpha}(t)\right|^{2} d t\right. \\
& \left.\quad+\int_{S}^{T} \int_{t}^{T}\left|f_{\alpha}\left(t, s, Y_{\alpha}(s), Z_{\alpha}(t, s), Z_{\alpha}(s, t)\right)-\bar{f}_{\alpha}\left(t, s, Y_{\alpha}(s), Z_{\alpha}(t, s), Z_{\alpha}(s, t)\right)\right|^{2} d s d t\right\} .
\end{aligned}
$$

In the sequel, we study the properties of continuity and differentiability of solutions to BSVIEs depending on a parameter. For notational convenience, for fixed $\alpha_{0}$, we write $\left(Y_{0}(\cdot), Z_{0}(\cdot, \cdot)\right)$ for $\left(Y_{\alpha_{0}}(\cdot), Z_{\alpha_{0}}(\cdot, \cdot)\right)$. Let us make the following assumptions:

(A3) The function $\alpha \rightarrow\left(f_{\alpha}, \psi_{\alpha}\right)$ is continuous; i.e., for any $\alpha_{0}$, $f_{\alpha}\left(t, s, Y_{0}(s), Z_{0}(t, s), Z_{0}(s, t)\right)-f_{\alpha_{0}}\left(t, s, Y_{0}(s), Z_{0}(t, s), Z_{0}(s, t)\right)$ converges to 0 in $L^{2}\left(0, T ; L_{\mathbb{F}}^{2}\left(\Omega \times(0, T) ; \mathbb{R}^{n}\right)\right)$ and $\psi_{\alpha}-\psi_{\alpha_{0}}$ converges to 0 in $C_{\mathbb{F}}\left(\Omega ; L^{2}\left(0, T ; \mathbb{R}^{n}\right)\right)$ as $\alpha \rightarrow \alpha_{0}$.

(A4) For any $\alpha \in \mathbb{R}, f_{\alpha}$ is differentiable with respect to $(y, z, v)$ with uniformly bounded derivatives denoted by $\partial_{y} f_{\alpha}, \partial_{z} f_{\alpha}$ and $\partial_{v} f_{\alpha}$ which are uniformly continuous; i.e., for any $\varepsilon>0$, there exists $\delta$ such that, for any $t, s \in[0, T], y \in \mathbb{R}^{n}, z, v \in \mathbb{R}^{n \times d}$,

$$
\left|\partial_{y} f_{\alpha}(t, s, y+h, z, v)-\partial_{y} f_{\alpha}(t, s, y, z, v)\right|<\varepsilon, \quad|h| \leq \delta,
$$

and the same holds for $z, v$ parts and for $\partial_{z} f_{\alpha}, \partial_{v} f_{\alpha}$.

(A5) The function $\alpha \mapsto\left(f_{\alpha}, \psi_{\alpha}\right)$ is differentiable; i.e., for any $\alpha_{0}$, the functions $\alpha \mapsto \psi_{\alpha}(t), \mathbb{R} \rightarrow C_{\mathbb{F}}\left(\Omega ; L^{2}\left(0, T ; \mathbb{R}^{n}\right)\right)$ and $\alpha \mapsto f_{\alpha}\left(t, s, Y_{0}(s), Z_{0}(t, s), Z_{0}(s, t)\right)$, $\mathbb{R} \rightarrow L^{2}\left(0, T ; L_{\mathbb{F}}^{2}\left(\Omega \times(0, T) ; \mathbb{R}^{n}\right)\right)$ are differentiable at $\alpha_{0}$ with derivatives $\partial_{\alpha} f_{\alpha_{0}}\left(t, s, Y_{0}(s), Z_{0}(t, s), Z_{0}(s, t)\right)$ and $\partial_{\alpha} \psi_{\alpha_{0}}(t)$.

Theorem 3.3 Let the coefficients $f_{\alpha}, \psi_{\alpha}(\alpha \in \mathbb{R})$ of BSVIEs (1.2) satisfy assumption (A2), and $\left(Y_{\alpha}(\cdot), Z_{\alpha}(\cdot, \cdot)\right)$ be solutions.

1. Suppose that $f_{\alpha}, \psi_{\alpha}$ satisfy assumption (A3). Then the function $\alpha \mapsto\left(Y_{\alpha}(\cdot), Z_{\alpha}(\cdot, \cdot)\right)$, $\mathbb{R} \rightarrow \mathcal{H}^{2}[0, T]$ is continuous.

2. Suppose that $f_{\alpha}, \psi_{\alpha}$ satisfy assumptions (A4)-(A5). Then the function $\alpha \mapsto\left(Y_{\alpha}(\cdot), Z_{\alpha}(\cdot, \cdot)\right), \mathbb{R} \rightarrow \mathcal{H}^{2}[0, T]$ is differentiable with derivatives given by $\left(\partial_{\alpha} Y_{\alpha}(\cdot), \partial_{\alpha} Z_{\alpha}(\cdot, \cdot)\right)$, which is the solution to the following BSVIE:

$$
\begin{aligned}
\partial_{\alpha} Y_{\alpha}(t)= & \partial_{\alpha} \psi_{\alpha}(t)+\int_{t}^{T}\left[\partial_{y} f_{\alpha}\left(t, s, Y_{\alpha}(s), Z_{\alpha}(t, s), Z_{\alpha}(s, t)\right) \partial_{\alpha} Y_{\alpha}(s)\right. \\
& +\left\langle\partial_{z} f_{\alpha}\left(t, s, Y_{\alpha}(s), Z_{\alpha}(t, s), Z_{\alpha}(s, t)\right), \partial_{\alpha} Z_{\alpha}(t, s)\right\rangle \\
& +\left\langle\partial_{\nu} f_{\alpha}\left(t, s, Y_{\alpha}(s), Z_{\alpha}(t, s), Z_{\alpha}(s, t)\right), \partial_{\alpha} Z_{\alpha}(s, t)\right\rangle \\
& \left.+\partial_{\alpha} f_{\alpha}\left(t, s, Y_{\alpha}(s), Z_{\alpha}(t, s), Z_{\alpha}(s, t)\right)\right] d s \\
& +\int_{t}^{T} \partial_{\alpha} Z_{\alpha}(t, s) d W(s), \quad t \in[0, T]
\end{aligned}
$$

where $\left\langle\partial_{z} f_{\alpha}, \partial_{\alpha} Z_{\alpha}\right\rangle \equiv\left(\partial_{z_{i}} f_{\alpha} \partial_{\alpha} Z_{\alpha}^{i}\right)_{1 \leq i \leq d}$ and $\left\langle\partial_{v} f_{\alpha}, \partial_{\alpha} Z_{\alpha}\right\rangle \equiv\left(\partial_{v_{i}} f_{\alpha} \partial_{\alpha} Z_{\alpha}^{i}\right)_{1 \leq i \leq d}$. 
Proof Property 1 is an immediate consequence of Theorem 3.2. Now, let us prove property 2 .

For simplicity, we can assume that $\alpha_{0}=0$ and $m=d=1$. For any $\alpha \neq 0$, put

$$
\Delta_{\alpha} Y(\cdot) \equiv \frac{Y_{\alpha}(\cdot)-Y_{0}(\cdot)}{\alpha}, \quad \Delta_{\alpha} Z(\cdot, \cdot) \equiv \frac{Z_{\alpha}(\cdot, \cdot)-Z_{0}(\cdot, \cdot)}{\alpha}
$$

Then

$$
\begin{aligned}
\Delta_{\alpha} Y(t)= & \frac{\psi_{\alpha}(t)-\psi_{0}(t)}{\alpha} \\
& +\int_{t}^{T} \frac{f_{\alpha}\left(t, s, Y_{\alpha}(s), Z_{\alpha}(t, s), Z_{\alpha}(s, t)\right)-f_{0}\left(t, s, Y_{0}(s), Z_{0}(t, s), Z_{0}(s, t)\right)}{\alpha} d s \\
& +\int_{t}^{T} \Delta_{\alpha} Z(t, s) d W(s), \quad t \in[0, T] .
\end{aligned}
$$

For any $\alpha \neq 0$, define

$$
\begin{aligned}
& A_{\alpha}(t, s) \equiv \begin{cases}\frac{f_{\alpha}\left(t, s, Y_{\alpha}(s), Z_{\alpha}(t, s), Z_{\alpha}(s, t)\right)-f_{\alpha}\left(t, s, Y_{0}(s), Z_{\alpha}(t, s), Z_{\alpha}(s, t)\right)}{Y_{\alpha}(s)-Y_{0}(s)}, & Y_{\alpha}(s) \neq Y_{0}(s), \\
\partial_{y} f_{\alpha}\left(t, s, Y_{0}(s), Z_{\alpha}(t, s), Z_{\alpha}(s, t)\right), & Y_{\alpha}(s)=Y_{0}(s),\end{cases} \\
& B_{\alpha}(t, s) \equiv \begin{cases}\frac{f_{\alpha}\left(t, s, Y_{0}(s), Z_{\alpha}(t, s), Z_{\alpha}(s, t)\right)-f_{\alpha}\left(t, s, Y_{0}(s), Z_{0}(t, s), Z_{\alpha}(s, t)\right)}{Z_{\alpha}(t, s)-Z_{0}(t, s)}, & Z_{\alpha}(t, s) \neq Z_{0}(t, s), \\
\partial_{z} f_{\alpha}\left(t, s, Y_{0}(s), Z_{0}(t, s), Z_{\alpha}(s, t)\right), & Z_{\alpha}(t, s)=Z_{0}(t, s),\end{cases} \\
& C_{\alpha}(t, s) \equiv \begin{cases}\frac{f_{\alpha}\left(t, s, Y_{0}(s), Z_{0}(t, s), Z_{\alpha}(s, t)\right)-f_{\alpha}\left(t, s, Y_{0}(s), Z_{0}(t, s), Z_{0}(s, t)\right)}{Z_{\alpha}(s, t)-Z_{0}(s, t)}, & Z_{\alpha}(s, t) \neq Z_{0}(s, t), \\
\partial_{v} f_{\alpha}\left(t, s, Y_{0}(s), Z_{0}(t, s), Z_{0}(s, t)\right), & Z_{\alpha}(s, t)=Z_{0}(s, t),\end{cases}
\end{aligned}
$$

and

$$
h_{\alpha}(t, s) \equiv \frac{f_{\alpha}\left(t, s, Y_{0}(s), Z_{0}(t, s), Z_{0}(s, t)\right)-f_{0}\left(t, s, Y_{0}(s), Z_{0}(t, s), Z_{0}(s, t)\right)}{\alpha}
$$

By virtue of $A_{\alpha}(\cdot, \cdot), B_{\alpha}(\cdot, \cdot), B_{\alpha}(\cdot, \cdot), h_{\alpha}(\cdot, \cdot)$, we can define

$$
F_{\alpha}(t, s, y, z, v) \equiv A_{\alpha}(t, s) y+B_{\alpha}(t, s) z+C_{\alpha}(t, s) v+h_{\alpha}(t, s) .
$$

Hence, BSVIE (3.2) can be written as

$$
\begin{aligned}
\Delta_{\alpha} Y(t)= & \frac{\psi_{\alpha}(t)-\psi_{0}(t)}{\alpha}+\int_{t}^{T} F_{\alpha}\left(t, s, \Delta_{\alpha} Y(s), \Delta_{\alpha} Z(t, s), \Delta_{\alpha} Z(s, t)\right) d s \\
& +\int_{t}^{T} \Delta_{\alpha} Z(t, s) d W(s), \quad t \in[0, T] .
\end{aligned}
$$

On the other hand, denote

$$
\begin{aligned}
F_{0}(t, s, y, z, v) \equiv & \partial_{y} f_{0}\left(t, s, Y_{0}(s), Z_{0}(t, s), Z_{0}(s, t)\right) y+\partial_{z} f_{0}\left(t, s, Y_{0}(s), Z_{0}(t, s), Z_{0}(s, t)\right) z \\
& +\partial_{v} f_{0}\left(t, s, Y_{0}(s), Z_{0}(t, s), Z_{0}(s, t)\right) v+\partial_{\alpha} f_{0}\left(t, s, Y_{0}(s), Z_{0}(t, s), Z_{0}(s, t)\right),
\end{aligned}
$$


and introduce the following BSVIE:

$$
\begin{aligned}
\partial_{\alpha} Y_{0}(t)= & \partial_{\alpha} \psi_{0}(t)+\int_{t}^{T} F_{0}\left(t, s, \partial_{\alpha} Y_{0}(s), \partial_{\alpha} Z_{0}(t, s), \partial_{\alpha} Z_{0}(s, t)\right) d s \\
& +\int_{t}^{T} \partial_{\alpha} Z_{0}(t, s) d W(s), \quad t \in[0, T] .
\end{aligned}
$$

By property 1 of this theorem, we know that $\left(Y_{\alpha}(\cdot), Z_{\alpha}(\cdot, \cdot)\right)$ converges to $\left(Y_{0}(\cdot), Z_{0}(\cdot, \cdot)\right)$ in $\mathcal{H}^{2}[0, T]$. We now have to prove that $\left(\Delta_{\alpha} Y(\cdot), \Delta_{\alpha} X(\cdot, \cdot)\right)$, the solution to BSVIE (3.3), converges to $\left(\partial_{\alpha} Y(\cdot), \partial_{\alpha} Z(\cdot, \cdot)\right)$, the solution to BSVIE (3.4), in $\mathcal{H}^{2}[0, T]$, as $\alpha$ tends to 0 . Also by property 1 , it is sufficient to check

$$
\begin{aligned}
& F_{\alpha}\left(t, s, \partial_{\alpha} Y_{0}(s), \partial_{\alpha} Z_{0}(t, s), \partial_{\alpha} Z_{0}(s, t)\right) \longrightarrow F_{0}\left(t, s, \partial_{\alpha} Y_{0}(s), \partial_{\alpha} Z_{0}(t, s), \partial_{\alpha} Z_{0}(s, t)\right) \\
& \quad \text { in } L^{2}\left(0, T ; L_{\mathbb{F}}^{2}(\Omega \times(0, T))\right)
\end{aligned}
$$

and

$$
\frac{\psi_{\alpha}(\cdot)-\psi_{0}(\cdot)}{\alpha} \longrightarrow \partial_{\alpha} \psi_{0}(\cdot) \quad \text { in } L_{\mathbb{F}}^{2}(\Omega \times(0, T))
$$

Equation (3.6) can be gotten by assumption (A5). Now, we check (3.5).

Notice that

$$
A_{\alpha}(t, s)=\int_{0}^{1} \partial_{y} f_{\alpha}\left(t, s, Y_{0}(s)+\lambda\left(Y_{\alpha}(s)-Y_{0}(s)\right), Z_{\alpha}(t, s), Z_{\alpha}(s, t)\right) d \lambda .
$$

Consequently,

$$
\begin{aligned}
\mathbb{E} \int_{0}^{T} & \int_{0}^{T}\left|A_{\alpha}(t, s)-\partial_{y} f_{\alpha}\left(t, s, Y_{0}(s), Z_{\alpha}(t, s), Z_{\alpha}(s, t)\right)\right|^{2}\left|\partial_{\alpha} Y_{0}(s)\right|^{2} d s d t \\
= & \mathbb{E} \int_{0}^{T} \int_{0}^{T} \mid \int_{0}^{1} \partial_{y} f_{\alpha}\left(t, s, Y_{0}(s)+\lambda\left(Y_{\alpha}(s)-Y_{0}(s)\right), Z_{\alpha}(t, s), Z_{\alpha}(s, t)\right) d \lambda \\
& -\left.\partial_{y} f_{\alpha}\left(t, s, Y_{0}(s), Z_{\alpha}(t, s), Z_{\alpha}(s, t)\right)\right|^{2}\left|\partial_{\alpha} Y_{0}(s)\right|^{2} d s d t \\
\leq & \mathbb{E} \int_{0}^{T} \int_{0}^{T} \int_{0}^{1} \mid \partial_{y} f_{\alpha}\left(t, s, Y_{0}(s)+\lambda\left(Y_{\alpha}(s)-Y_{0}(s)\right), Z_{\alpha}(t, s), Z_{\alpha}(s, t)\right) \\
& -\left.\partial_{y} f_{\alpha}\left(t, s, Y_{0}(s), Z_{\alpha}(t, s), Z_{\alpha}(s, t)\right)\right|^{2}\left|\partial_{\alpha} Y_{0}(s)\right|^{2} d \lambda d s d t .
\end{aligned}
$$

Set $\Omega_{1}=\left\{\left|Y_{\alpha}(s)-Y_{0}(s)\right| \leq \delta\right\}$. Hence, by assumption (A4),

$$
\begin{aligned}
\mathbb{E} \int_{0}^{T} & \int_{0}^{T} \int_{0}^{1} \mid \partial_{y} f_{\alpha}\left(t, s, Y_{0}(s)+\lambda\left(Y_{\alpha}(s)-Y_{0}(s)\right), Z_{\alpha}(t, s), Z_{\alpha}(s, t)\right) \\
& -\left.\partial_{y} f_{\alpha}\left(t, s, Y_{0}(s), Z_{\alpha}(t, s), Z_{\alpha}(s, t)\right)\right|^{2}\left|\partial_{\alpha} Y_{0}(s)\right|^{2} d \lambda d s d t \\
\leq & \mathbb{E} \int_{0}^{T} \int_{0}^{T}\left[\varepsilon^{2}\left|\partial_{\alpha} Y_{0}(s)\right|^{2}+2 L^{2} \chi_{\Omega_{1}^{c}}\left|\partial_{\alpha} Y_{0}(s)\right|^{2}\right] d s d t \\
\quad \leq & \varepsilon^{2} T \mathbb{E} \int_{0}^{T}\left|\partial_{\alpha} Y_{0}(s)\right|^{2} d s+2 L^{2} T \mathbb{E} \int_{0}^{T} \chi_{\Omega_{1}^{c}}\left|\partial_{\alpha} Y_{0}(s)\right|^{2} d s .
\end{aligned}
$$


Now, we estimate the second term on the right side of the above inequality. For given $M>0$, define $\Omega_{2}=\left\{\left|\partial_{\alpha} Y_{0}(s)\right| \leq M\right\}$. Then

$$
\begin{aligned}
& \mathbb{E} \int_{0}^{T} \chi_{\Omega_{1}^{c}}\left|\partial_{\alpha} Y_{0}(s)\right|^{2} d s \\
& \quad=\mathbb{E} \int_{0}^{T} \chi_{\Omega_{1}^{c}} \chi_{\Omega_{2}}\left|\partial_{\alpha} Y_{0}(s)\right|^{2} d s+\mathbb{E} \int_{0}^{T} \chi_{\Omega_{1}^{c}} \chi_{\Omega_{2}^{c}}\left|\partial_{\alpha} Y_{0}(s)\right|^{2} d s \\
& \quad \leq \mathbb{E} \int_{0}^{T} \chi_{\Omega_{1}^{c}} \chi_{\Omega_{2}} M^{2} \frac{\left|Y_{\alpha}(s)-Y_{0}(s)\right|^{2}}{\delta^{2}} d s+\mathbb{E} \int_{0}^{T} \chi_{\Omega_{2}^{c}}\left|\partial_{\alpha} Y_{0}(s)\right|^{2} d s \\
& \quad \leq \frac{M^{2}}{\delta^{2}} \mathbb{E} \int_{0}^{T}\left|Y_{\alpha}(s)-Y_{0}(s)\right|^{2} d s+\mathbb{E} \int_{0}^{T} \chi_{\left\{\left|\partial_{\alpha} Y_{0}(s)\right|>M\right\}}\left|\partial_{\alpha} Y_{0}(s)\right|^{2} d s .
\end{aligned}
$$

By the Lebesgue dominated convergence theorem, since $\partial_{\alpha} Y_{0}(\cdot) \in L_{\mathbb{F}}^{2}(\Omega \times(0, T))$, one deduces that

$$
\mathbb{E} \int_{0}^{T} \chi_{\left\{\left|\partial_{\alpha} Y_{0}(s)\right|>M\right\}}\left|\partial_{\alpha} Y_{0}(s)\right|^{2} d s \rightarrow 0
$$

By property $1, Y_{\alpha}(\cdot) \rightarrow Y_{0}(\cdot)$ in $L_{\mathbb{F}}^{2}(\Omega \times(0, T))$, one gets

$$
\frac{M^{2}}{\delta^{2}} \mathbb{E} \int_{0}^{T}\left|Y_{\alpha}(s)-Y_{0}(s)\right|^{2} d s \rightarrow 0
$$

Equation (3.7), together with (3.8)-(3.11), yields

$$
\mathbb{E} \int_{0}^{T} \int_{0}^{T}\left|A_{\alpha}(t, s)-\partial_{y} f_{\alpha}\left(t, s, Y_{0}(s), Z_{\alpha}(t, s), Z_{\alpha}(s, t)\right)\right|^{2}\left|\partial_{\alpha} Y_{0}(s)\right|^{2} d s d t \rightarrow 0 .
$$

Denote $\Omega_{3}=\left\{\left|Z_{\alpha}(t, s)-Z_{0}(t, s)\right| \leq \delta\right\}$. Then

$$
\begin{aligned}
\mathbb{E} \int_{0}^{T} & \int_{0}^{T} \mid \partial_{y} f_{\alpha}\left(t, s, Y_{0}(s), Z_{\alpha}(t, s), Z_{\alpha}(s, t)\right) \\
& -\left.\partial_{y} f_{\alpha}\left(t, s, Y_{0}(s), Z_{0}(t, s), Z_{\alpha}(s, t)\right)\right|^{2}\left|\partial_{\alpha} Y_{0}(s)\right|^{2} d s d t \\
\leq & \varepsilon^{2} T \mathbb{E} \int_{0}^{T}\left|\partial_{\alpha} Y_{0}(s)\right|^{2} d s+L^{2} \mathbb{E} \int_{0}^{T} \int_{0}^{T} \chi_{\Omega_{3}^{c}}\left|\partial_{\alpha} Y_{0}(s)\right|^{2} d s d t \\
\leq & \varepsilon^{2} T \mathbb{E} \int_{0}^{T}\left|\partial_{\alpha} Y_{0}(s)\right|^{2} d s+\frac{L^{2} M^{2}}{\delta^{2}} \mathbb{E} \int_{0}^{T} \int_{0}^{T}\left|Z_{\alpha}(t, s)-Z_{0}(t, s)\right|^{2} d s d t \\
\quad & L^{2} T \mathbb{E} \int_{0}^{T} \chi_{\left\{\left|\partial_{\alpha} Y_{0}(s)\right|>M\right\}}\left|\partial_{\alpha} Y_{0}(s)\right|^{2} d s \\
\rightarrow & 0 .
\end{aligned}
$$

Similarly, we can check that

$$
\begin{aligned}
& \mathbb{E} \int_{0}^{T} \int_{0}^{T} \mid \partial_{y} f_{\alpha}\left(t, s, Y_{0}(s), Z_{0}(t, s), Z_{\alpha}(s, t)\right) \\
& \quad-\left.\partial_{y} f_{\alpha}\left(t, s, Y_{0}(s), Z_{0}(t, s), Z_{0}(s, t)\right)\right|^{2}\left|\partial_{\alpha} Y_{0}(s)\right|^{2} d s d t \rightarrow 0 .
\end{aligned}
$$


Combining (3.12) with (3.13), (3.14), we can obtain

$$
\mathbb{E} \int_{0}^{T} \int_{0}^{T}\left|A_{\alpha}(t, s)-\partial_{y} f_{\alpha}\left(t, s, Y_{0}(s), Z_{0}(t, s), Z_{0}(s, t)\right)\right|^{2}\left|\partial_{\alpha} Y_{0}(s)\right|^{2} d s d t \rightarrow 0
$$

With a similar procedure, we can get

$$
\begin{aligned}
& \mathbb{E} \int_{0}^{T} \int_{0}^{T}\left|B_{\alpha}(t, s)-\partial_{z} f_{\alpha}\left(t, s, Y_{0}(s), Z_{0}(t, s), Z_{0}(s, t)\right)\right|^{2}\left|\partial_{\alpha} Z_{0}(t, s)\right|^{2} d s d t \rightarrow 0, \\
& \mathbb{E} \int_{0}^{T} \int_{0}^{T}\left|C_{\alpha}(t, s)-\partial_{v} f_{\alpha}\left(t, s, Y_{0}(s), Z_{0}(t, s), Z_{0}(s, t)\right)\right|^{2}\left|\partial_{\alpha} Z_{0}(s, t)\right|^{2} d s d t \rightarrow 0,
\end{aligned}
$$

and

$$
\mathbb{E} \int_{0}^{T} \int_{0}^{T}\left|h_{\alpha}(t, s)-\partial_{\alpha} f_{0}\left(t, s, Y_{0}(s), Z_{0}(t, s), Z_{0}(s, t)\right)\right|^{2} d s d t \rightarrow 0
$$

By (3.15)-(3.17), we prove (3.5). That completes the proof.

An immediate consequence of Theorem 3.3 is now the following.

Corollary 3.4 Suppose that $(Y(\cdot), Z(\cdot, \cdot))$ is the solution to BSVIE:

$$
\begin{aligned}
Y(t)= & \psi(X(t))+\int_{t}^{T} f(t, s, Y(s), Z(t, s), Z(s, t)) d s \\
& +\int_{t}^{T} Z(t, s) d W(s), \quad t \in[0, T]
\end{aligned}
$$

where $\psi(\cdot)$ is differentiable and $f(\cdot, \cdot, \cdot, \cdot, \cdot)$ is differentiable with respect to $(y, z, v)$ with uniformly bounded derivatives. Here X(.) solves SVIE (2.7) and the coefficients $b(\cdot, \cdot, \cdot), \sigma(\cdot, \cdot, \cdot)$ satisfy the assumptions in Corollary 2.6. Then $(\nabla Y(\cdot), \nabla Z(\cdot, \cdot))$ solves the following BSVIE:

$$
\begin{aligned}
\nabla Y(t)= & \partial_{x} \psi(X(t)) \nabla X(t)+\int_{t}^{T} \partial_{y} f(t, s, Y(s), Z(t, s), Z(s, t)) \nabla Y(s) \\
& +\left\langle\partial_{z} f(t, s, Y(s), Z(t, s), Z(s, t)), \nabla Z(t, s)\right\rangle \\
& +\left\langle\partial_{v} f(t, s, Y(s), Z(t, s), Z(s, t)), \nabla Z(s, t)\right\rangle d s \\
& +\int_{t}^{T} \nabla Z(t, s) d W(s), \quad t \in[0, T]
\end{aligned}
$$

\section{Conclusions}

In this paper, we consider the properties of continuity and differentiability of solutions to two kinds of stochastic Volterra integral equations: forward equations (Theorem 2.5) and backward equations (Theorem 3.3). As an application, we obtain the deeper wellposedness results on the structure of the solution to forward/backward stochastic Volterra integral equations (Corollaries 2.6 and 3.4). 


\section{Acknowledgements}

This work is supported in part by the National Natural Science Foundation of China (11526167), the Fundamental Research Funds for the Central Universities (XDJK2017C054), the Natural Science Foundation of CQCSTC (2015jcyjA00017) This work was carried out during the stay of the author at University of Central Florida, USA. The author would like to thank the Department of Mathematics for its hospitality, and the financial support from China Scholarship Council.

\section{Competing interests}

The author declares that he has no competing interests.

Author's contributions

Author read and approved the final manuscript.

\section{Publisher's Note}

Springer Nature remains neutral with regard to jurisdictional claims in published maps and institutional affiliations.

Received: 3 April 2017 Accepted: 9 October 2017 Published online: 17 October 2017

\section{References}

1. Bichteler, K: Stochastic Integration with Jumps. Encyclopedia of Mathematics and Its Applications, vol. 89. Cambridge University Press, Cambridge (2002)

2. Friedman, A: Stochastic Differential Equations and Applications, Vol. 1. Probability and Mathematical Statistics, vol. 28. Academic Press, New York (1975)

3. Gihman, II, Skorohod, AV: Stochastic Differential Equations. Ergebnisse der Mathematik und ihrer Grenzgebiete, vol. 72. Springer, New York (1972). Translated from the Russian by Kenneth Wickwire

4. Stroock, DW: Lectures on Topics in Stochastic Differential Equations. Tata Institute of Fundamental Research Lectures on Mathematics and Physics, Tata Institute of Fundamental Research, Bombay, vol. 68. Springer, Berlin (1982). With notes by Satyajit Karmakar

5. Chen, S, Yong, J: A linear quadratic optimal control problem for stochastic Volterra integral equations. In: Control Theory and Related Topics, pp. 44-66. World Scientific, Hackensack (2007)

6. Wang, T, Shi, Y: Linear quadratic stochastic integral games and related topics. Sci. China Math. 58, 2405-2420 (2015)

7. Berger, MA, Mizel, VJ: Volterra equations with Itô integrals. I. J. Integral Equ. 2, 187-245 (1980)

8. Berger, MA, Mizel, VJ: Volterra equations with Itô integrals. II. J. Integral Equ. 2, 319-337 (1980)

9. Ito, I: On the existence and uniqueness of solutions of stochastic integral equations of the Volterra type. Kodai Math. 2, 158-170 (1979)

10. Protter, P: Volterra equations driven by semimartingales. Ann. Probab. 13, 519-530 (1985)

11. Lin, J: Adapted solution of a backward stochastic nonlinear Volterra integral equation. Stoch. Anal. Appl. 20, 165-183 (2002)

12. Yong, J: Well-posedness and regularity of backward stochastic Volterra integral equations. Probab. Theory Relat. Fields 142, 21-77 (2008)

13. El Karoui, N, Peng, S, Quenez, MC: Backward stochastic differential equations in finance. Math. Finance 7, 1-71 (1997)

14. Kromer, E, Overbeck, L: Differentiability of BSVIEs and dynamic capital allocations. Preprint (2015)

\section{Submit your manuscript to a SpringerOpen ${ }^{\circ}$ journal and benefit from:}

- Convenient online submission

$\checkmark$ Rigorous peer review

- Open access: articles freely available online

- High visibility within the field

Retaining the copyright to your article

Submit your next manuscript at $\boldsymbol{~ s p r i n g e r o p e n . c o m ~}$ 